\title{
BMJ Open Barriers and facilitators to learn and improve through morbidity and mortality conferences: a qualitative study
}

Marit S de Vos, ${ }^{1,2}$ Jaap F Hamming, ${ }^{1}$ Perla J Marang-van de Mheen $^{2}$

To cite: de Vos MS, Hamming JF, Marang-van de Mheen PJ. Barriers and facilitators to learn and improve through morbidity and mortality conferences: a qualitative study. BMJ Open

2017;7:e018833. doi:10.1136/ bmjopen-2017-018833

- Prepublication history and additional material for this paper are available online. To view these files, please visit the journal online (http://dx.doi. org/10.1136/bmjopen-2017018833).

Received 25 July 2017 Revised 6 September 2017 Accepted 28 September 2017

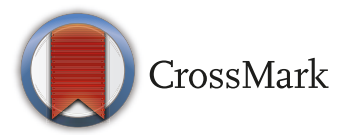

${ }^{1}$ Department of Surgery, Leiden University Medical Centre, Leiden, The Netherlands ${ }^{2}$ Department of Medical Decision Making, Leiden University Medical Centre, Leiden, The Netherlands

Correspondence to

Marit S de Vos;

m.s.de_vos@lumc.nl

\section{ABSTRACT}

Objectives To explore barriers and facilitators to successful morbidity and mortality conferences (M\&M), driving learning and improvement.

Design This is a qualitative study with semistructured interviews. Inductive, thematic content analysis was used to identify barriers and facilitators, which were structured across a pre-existing framework for change in healthcare. Setting Dutch academic surgical department with a long tradition of M\&M.

Participants An interview sample of surgeons, residents and physician assistants $(\mathrm{n}=12)$.

Results A total of 57 barriers and facilitators to successful $M \& M$, covering 18 themes, varying from 'case type' to 'Ieadership', were perceived by surgical staff. While some factors related to M\&M organisation, others concerned individual or social aspects. Eight factors, of which four were at the social level, had simultaneous positive and negative effects (eg, 'hierarchy' and 'team spirit'). Mediating pathways for M\&M success were found to relate to available information, staff motivation and realisation processes.

Conclusions This study provides leads for improvement of M\&M practice, as well as for further research on key elements of successful M\&M. Various factors were perceived to affect M\&M success, of which many were individual and social rather than organisational factors, affecting information and realisation processes but also staff motivation. Based on these findings, practical recommendations were formulated to guide efforts towards best practices for M\&M.

\section{INTRODUCTION}

The morbidity and mortality conference $(\mathrm{M} \& \mathrm{M})$ is a deep-rooted tradition in surgery, adopted by many other medical specialties, aiming to serve both educational and quality improvement (QI) purposes. ${ }^{12} \mathrm{M} \& \mathrm{M}$ additionally provides opportunities to teach principles of patient safety and QI, which are current requirements for residency education. $^{3-5}$ Despite similar objectives, significant variation exists in M\&M practice. ${ }^{13}$ Case presentations and discussions may highlight important learning points, but
Strengths and limitations of this study

This is the first qualitative study to assess success factors of morbidity and mortality conferences.

- Strengths of this study design include the use of purposive sampling and data saturation to obtain a diversity of viewpoints and increase the ability to identify all relevant factors.

- Because of the single-centre design, some findings may particularly be representative of teaching hospitals and surgical specialties.

implementation and follow-up often receive less attention at the conference, which is a known challenge for many improvement practices in healthcare..$^{5-9}$

M\&M practice variation is likely related to the fact that key factors for successful M\&M, driving learning and improvement, remain largely unclear. Factors that have been reported include organisational aspects, such as a structured approach to review events, ${ }^{1011}$ the use of moderators, ${ }^{2}{ }^{12-14}$ and participation of all involved staff, ${ }^{10} 1516$ which were corroborated by survey studies. ${ }^{3}{ }^{17-20}$ Except for the importance of a safe, blame-free environment, ${ }^{212}$ the impact of non-organisational factors, such as team dynamics, has not been considered. While learning and change theories stipulate that these processes occur at different levels, affected by various factors at the individual and team levels, ${ }^{21-24}$ it remains unknown to what extent these factors affect learning and improvement processes at M\&M.

We hypothesised that barriers and facilitators to successful M\&M, resulting in learning and improvement, also exist at the individual and social level. To obtain a broad and nuanced understanding of the complexity of factors influencing M\&M success, a qualitative approach was used. Qualitative studies have rarely been used to study M\&M, but 
can yield rich insights that may not be revealed by quantitative assessments. The purpose of this study was to enhance understanding of the barriers, facilitators and mediating pathways to successful M\&M, driving learning and improvement of clinical practice.

\section{METHODS}

A total of 12 semistructured 1-hour interviews were conducted to identify barriers and facilitators for successful M\&M. This qualitative approach was chosen as it allows exploring perceptions, and encourages participants to share rich descriptions and in-depth information. ${ }^{25}$ The number of 12 interviews was selected because of feasibility and anticipated number needed to reach data saturation, defined as three consecutive interviews without additional themes emerging. ${ }^{26}$ Purposive sampling was used to invite participants via telephone or email, varying gender, seniority and surgical subspecialty to obtain a diversity of viewpoints and hence increase the ability to identify all relevant barriers and facilitators. Standards for reporting qualitative research were used to guide reporting of this study. ${ }^{27}$

All invited agreed to participate, including six attending surgeons, five surgical residents and one physician assistant (PA) (four women; mean local work experience: 7.2 years (range 1-18 years)). All worked at the surgical department of a large academic hospital in the Netherlands (882 beds), covering general, endocrine, vascular, gastrointestinal, paediatric, oncological, trauma and transplant surgery (all represented in the interview sample). All interviewees had prior experience with M\&M practice at other, mostly teaching, hospitals. The department has a long tradition of departmental M\&M meetings, which gather all faculty, residents, PAs and medical students to discuss a single case during a 1-hour conference every 2 weeks. More details on the local M\&M format can be found in prior publications. ${ }^{28}{ }^{29}$ Cases are selected and presented by residents under faculty supervision (ie, regardless of their involvement). A single case is presented per meeting with the aid of a fixed presentation format, which is followed by a $20-40$ min discussion led by a moderator. ${ }^{29}$

Prior to the interview, participants were informed about the study objectives and design. Identity of interviewees was kept anonymous to both colleagues and department chiefs to protect confidentiality and promote openness. A topic guide was developed to guide the interviews (online supplementary appendix 1). First, participants were asked about their overall opinion on M\&M practice and what factors may affect M\&M success, defined as a conference that results in learning and improvement. This broad definition was intentionally selected to allow interviewees to freely explore what makes a successful M\&M. Interviewees were encouraged to discuss experiences with M\&M in both the local and other hospitals (eg, due to hospital rotation during residency), as well as factors that they expected but never experienced. Further questions related to the perceived effect of factors that are most common in the M\&M literature, which related to the conference's structure (ie, attendance, culture) and content (ie, case selection, presentation, moderation, deriving plans) ${ }^{329}$ Questions about experiences with the local M\&M were used to evoke discussion of generic success factors and barriers (eg, what illustrates that your M\&M is (not) free of shame and blame?).

Each interviewee was interviewed individually in a conference room of a research department in the hospital. Interviews were audio-taped and transcribed in full. Anonymised transcripts were analysed using thematic content analysis with an inductive, data-driven approach, which involved a recursive process of open coding and collocating codes into themes. ${ }^{30}$ Coding was performed on ATLAS.ti software (ATLAS.ti Scientific Software Development GmbH, Berlin, Germany) by the same researcher who individually conducted the interviews (MSdV). This researcher has an MD degree and experience in research on $\mathrm{M} \& \mathrm{M},{ }^{29}{ }^{32}$ but no professional relationship with interviewees as she is currently not involved in clinical work. A second coder, who was a research assistant with qualitative research experience, independently reviewed all coded transcripts for continuity of data interpretation and any miscoded statements, and discussed with the primary coder until consensus was reached. To guide the analysis, emerging themes were structured across six domains of a pre-existing framework for barriers to and incentives for change in healthcare, developed based on various theories and models for implementing change. ${ }^{22}$ Domains included case (adapted from 'patient'), action (adapted from 'innovation'), individual professional, social context, organisational context and external context. Frequencies of reported factors were only reported when notably high, low or different between residents and faculty. Factors were assessed for their direction of effect (ie, facilitator, barrier or both) and their pathways to achieve a successful M\&M (ie, how exactly does this enhance M\&M-based learning and improvement?). The mediating pathways for M\&M success identified in this study were subsequently assessed for their relation to existing, more general frameworks for improvement in healthcare. ${ }^{22}$

\section{RESULTS}

A total of 57 facilitators and barriers for M\&M success were reported by interviewed professionals (table 1). All were reported in at least three interviews, and data saturation was reached at the 10th interview. More facilitators than barriers were reported, with most facilitators at the case level and most barriers at the organisational level. Many facilitators could also serve as a barrier if absent or insufficient (eg, motivation), but for eight factors, of which four were at the social level, both positive and negative effects were perceived simultaneously (eg, hierarchy) (table 1). Illustrative quotes for all facilitators and barriers are provided in online supplementary appendix 
Table 1 Facilitators and barriers to successful M\&M practice, grouped in themes and structured across levels of a framework for achieving change in healthcare

\begin{tabular}{|c|c|c|c|}
\hline Theme & Factor & $\begin{array}{c}\text { Facilitator } \\
(+)\end{array}$ & $\begin{array}{c}\text { Barrier } \\
(-)\end{array}$ \\
\hline \multicolumn{4}{|l|}{ Case level } \\
\hline \multirow[t]{3}{*}{ Type of case (1) } & Attractive topic & + & \\
\hline & Clinical relevance & + & \\
\hline & $\begin{array}{l}\text { Value for education/ } \\
\text { improvement }\end{array}$ & + & \\
\hline \multirow[t]{6}{*}{ Information (2) } & Includes local data & + & \\
\hline & Literature & + & \\
\hline & Skills education & + & \\
\hline & $\begin{array}{l}\text { Information from those } \\
\text { involved }\end{array}$ & + & - \\
\hline & Addressing system factors & + & \\
\hline & Addressing 'soft skills' & + & \\
\hline \multirow[t]{4}{*}{ Presentation (3) } & Qualified presenter & + & \\
\hline & Proper preparation & + & \\
\hline & Proper supervision & + & \\
\hline & Fixed format & + & \\
\hline \multicolumn{4}{|l|}{ Action level } \\
\hline \multirow[t]{4}{*}{ Type of plan (4) } & Attractive topic & + & \\
\hline & Clinically significant topic & + & \\
\hline & More disciplines involved & & - \\
\hline & Higher complexity & & - \\
\hline \multirow[t]{4}{*}{ Planning (5) } & Explicitly formulated & + & \\
\hline & Responsibility assigned & + & - \\
\hline & Time frame determined & + & \\
\hline & Included in protocols & + & \\
\hline \multicolumn{4}{|l|}{ Individual level } \\
\hline \multirow[t]{4}{*}{ Motivation (6) } & Intrinsic motivation & + & \\
\hline & Interest in specific topic & + & \\
\hline & Values/beliefs & + & - \\
\hline & Other priorities/incentives & & - \\
\hline Participation (7) & Personality & + & - \\
\hline \multirow[t]{2}{*}{ Realisation (8) } & Empowerment, control & + & \\
\hline & Forgetfulness & & - \\
\hline
\end{tabular}

\section{Social level}

\begin{tabular}{llll} 
Culture (9) & Safe environment & + & \\
& Team spirit & + & - \\
Seadership (10) & Reinforcing attendance & + & - \\
& Reinforcing actions & + & \\
& Hierarchy & + & - \\
& Exemplary behaviour & + & \\
Participants (11) & Participation of experts & + & \\
& Interactivity & + & \\
& Audience composition/size & + & - \\
& Multidisciplinary & + & - \\
participation & & \\
\hline
\end{tabular}

Continued

\section{Table 1 Continued}

\begin{tabular}{|c|c|c|c|}
\hline Theme & Factor & $\begin{array}{l}\text { Facilitator } \\
\qquad(+)\end{array}$ & $\begin{array}{c}\text { Barrier } \\
(-)\end{array}$ \\
\hline \multicolumn{4}{|c|}{ Organisational level } \\
\hline \multirow[t]{6}{*}{ M\&M format (13) } & $\begin{array}{l}\text { Strong focus on } \\
\text { improvement }\end{array}$ & + & \\
\hline & M\&M in specialist setting & + & \\
\hline & $\begin{array}{l}\text { Communications (before/ } \\
\text { after) }\end{array}$ & + & \\
\hline & $\begin{array}{l}\text { Too many cases per } \\
\text { meeting }\end{array}$ & & - \\
\hline & No tracking of actions & & - \\
\hline & $\begin{array}{l}\text { No check/feedback on } \\
\text { effect }\end{array}$ & & - \\
\hline \multirow[t]{3}{*}{ Reporting (14) } & System for data collection & + & \\
\hline & Difficult access to data & & - \\
\hline & $\begin{array}{l}\text { Lack of feedback from } \\
\text { data }\end{array}$ & & - \\
\hline \multirow[t]{4}{*}{ Staff (15) } & Dedicated staff/committee & + & \\
\hline & Super specialisation & & - \\
\hline & Staff turnover & & - \\
\hline & $\begin{array}{l}\text { Other/conflicting } \\
\text { expectations }\end{array}$ & & - \\
\hline \multirow[t]{2}{*}{ Time (16) } & Overall lack of time & & - \\
\hline & Receiving dedicated time & + & \\
\hline \multicolumn{4}{|l|}{ External level } \\
\hline \multirow[t]{2}{*}{ Healthcare (17) } & Inevitability ('nature') & & - \\
\hline & Benchmarking & + & \\
\hline
\end{tabular}

M\&M, morbidity and mortality conference.

2. Facilitators and barriers were grouped into 17 themes, which will be discussed per level of the framework for change in healthcare (table 1).

\section{Case/action level}

The type of case discussed at M\&M as well as the type of action items were reported as influencing factors. Cases and actions dealing with clinically relevant and attractive topics (ie, high severity/frequency and surgical technical issues) were perceived to increase sense of urgency to bring about change (table 1 ).

We like that [surgical technique]. We're all very practical people. $(\# 7)$

To enhance information transfer, presenters should be skilful, well-prepared and supervised, using fixed presentation formats to cover the case, pertinent literature, surgical skills and involved system-level factors. M\&M was also seen as an important opportunity to address soft skills, such as communication or emotional impact. Including local data and trends was perceived to instigate reflection and increase the sense of urgency.

(...) about pneumonia, everyone will be like 'oh no, boring', but if you present a concise plan and numbers and those things, then, I think that'd be very nice, because that concerns everyone. (\#5) 
Details regarding context and deliberations in cases should be obtained from those involved, but some residents added that (emotional) involvement might also bias judgement and hinder information accuracy.

Overall complexity of proposed actions was perceived as a barrier to implementation and considered to increase with the number of people or disciplines involved. Hence plans should be explicit, including a timeline and person in charge. At the same time, however, top-down task assignment could hinder implementation, referred to as 'mandatory volunteerism'.

If you just send someone off like 'you go do that', that won't work, it has been proven. (\#9)

\section{Individual level}

In various ways, professionals perceived 'motivation' as a powerful and important facilitator for M\&M, enhancing attendance rates, active participation and subsequent realisation of actions (table 1 ). Motivation was considered to improve when M\&M covered topics applicable to one's own practice or field of interest, or when topics were accompanied by a sense of urgency.

Individual personalities were mentioned as potential facilitators as well as barriers, as for example insecurity may hamper speaking up, while other personality traits could be beneficial in that respect. Similarly, personal values and beliefs could enhance or impede motivation to attend, participate, and carry out actions. Feedback on actions from prior conferences was considered essential to demonstrate the value of M\&M.

Did anything change? (...) Feedback needs to improve greatly, otherwise it's so useless. (\#10)

A barrier was perceived in that staff may prioritise other activities over M\&M, such as clinical work or training duties (mostly mentioned by residents) or subspecialty-related activities (mostly mentioned by faculty).

I'm particularly interested in my own service [ie, subspecialty], those are my patients and my trainees. (\#6)

Some noted that it should be prevented that M\&M is considered a 'chore' as this decreases motivation, but others considered such 'chores' components of professionalism.

(...) some things are chores, but just need to be done. (\#4)

\section{Social level}

The need for a safe environment to allow for an open discussion was often expressed (table 1). In this respect, a strong sense of team spirit was considered beneficial (eg, counting on support from peers), but also a potential barrier as one may withhold comments to avoid offending a colleague, referred to as 'back-stabbing' (online supplementary appendix 2). Super specialisation in surgery was mentioned by all but one interviewee, and considered to have negatively affected team spirit, decreasing interest and motivation for topics outside one's subspecialty.

If you talk about pseudarthrosis, I'm sure no gastrointestinal or vascular guy really enjoys it. (\#5)

Some suggested that M\&M could therefore cover more general topics or increasingly focus on more general aspects, such as communication skills or teamwork involved, as these are shared among different subspecialties.

Leadership was assigned a critical role in harnessing this desired culture through exemplary behaviour and actively lowering barriers to speaking up.

It helps to see that things at times go wrong even for someone you perhaps admire, some expert. (\#11)

Some believed that faculty attendance may set an example to juniors, but others believed that mandatory attendance should be actively reinforced with staff held accountable for absences. All stressed that leadership should check and reinforce progress of M\&M-derived actions, and that hierarchy helps in this respect. At the same time, hierarchy may serve as a barrier to an open discussion.

If you really want to promote free speech, then faculty should emphasize that hierarchy is put aside during such a conference. (\#7)

To steer discussions, promoting a safe atmosphere, the use of moderators was considered helpful.

While high attendance rates may serve as a motivator and increase available information and reach, a smaller audience size may better promote a safe and open environment. Similarly, audience composition (ie, who is present) can both positively and negatively affect the discussion.

You really think about who is involved and try to predict how that person will respond. In some cases, you'll decide: well, I'm not going to do that here. (\#3)

Specifically, it was considered important to increase interactivity and involve experts or staff who had been involved in the cases, to enhance discussion quality and participant experience. Multidisciplinary participation was considered to provide essential information, but also to potentially negatively affect openness and level of discussions.

Well then there might be some competence differences. Perhaps for some topics it could work, but not in general I'd say. (\#9)

\section{Organisational/external level}

With regard to the M\&M format, a strong focus on improvement and (preceding) communications was considered beneficial. Handling too many cases was mentioned as a potential barrier, as it may decrease attention and time 
for discussing opportunities for improvement (table 1). With regard to the setting, most faculty (4 of 6 ) advocated for subspecialty rather than departmental M\&M, as it would allow discussions to focus on subspecialist topics, which would increase participants' motivation and ability to change processes at their own ward. Moreover, super specialisation may currently limit one's ability to attend M\&M.

My weeks are overloaded with duties related to my subspecialty (...) An unstoppable phenomenon. The generic conferences suffer from it. (\#4)

Reporting systems were appreciated for their value to collect local data, but lack of feedback was considered a missed opportunity to increase sense of urgency for topics and encourage reporting behaviour. Residents currently perceived a barrier in that it was too labour-intensive and difficult to access local data, while this could provide essential support for case selection, presentations and follow-up. Many also missed systematic follow-up, evaluation and feedback on prior actions at M\&M.

A sort of follow-up makes it all more cohesive, of course, it'll give you the feeling that you're all involved in a sort of improvement cycle rather than scattershot. (\#8)

Lack of continuity due to typical staff turnover in teaching hospitals was considered to hamper (sustaining) improvements.

With varying doctors and trainees, you simply need to repeat things. (...) another group arrives from another hospital, with a different standard practice, where they were used to do things differently. (\#9)

It was suggested, mostly by faculty, to assign dedicated staff to monitor outcome data and implement plans for improvement.

(...) in task forces because they'll put it on their agenda and have something to say about that topic, about quality. (\#11)

General lack of time was mentioned in all but one interview, as an important barrier to preparation, attendance and realisation of actions. Similarly, staff may face too many, sometimes conflicting, expectations.

We expect single individuals to fulfil all these requirements for clinical practice, research, training, leadership ánd management (...) that's thé inhibiting factor! Too many tasks and too many different tasks. (\#2)

Receiving dedicated time to work on tasks arising from M\&M was perceived to facilitate these processes.

We rather do it at night to avoid missing surgeries, clinic or clinical...that's the focus of our training, clinical practice. (...) If we decide, and acknowledge [that M\&M is of equal importance], then I think that
Table 2 Mediating pathways for M\&M-based learning and improving that are affected by reported facilitators and barriers
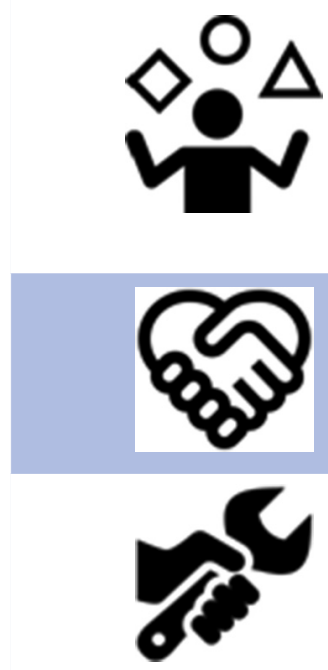

INFORMATION (to know), that is, complete/clear/ accessible information, presentations, data/ trends, communications, feedback, input/discussion, dissemination

MOTIVATION (to want), that is, participant attendance, participation, experience, engagement, support, sense of urgency

REALISATION (to can/

do), that is, ensure a clear objective and extensive plan, feasibility, empowerment for change, follow-up/tracking, (re)evaluation, sustaining

M\&M, morbidity and mortality conference.

we should organize it in such a way that residents receive half a day to do these things. (\#7)

Only two external-level factors were reported: the 'nature' of healthcare, balancing risks and benefits (eg, haemorrhage and thrombosis prevention) was perceived to prevent complete eradication of adverse events, and benchmarking local performance against other centres was often mentioned as an important facilitator, serving as a source of information and motivator.

\section{Pathways to M\&M success}

The reported facilitators and barriers appeared to enhance or impede the following:

1. Whether professionals are adequately informed to identify targets and plans for improvement.

2. Whether professionals are motivated to participate in, and support, M\&M practice and the ensuing actions.

3. Whether professionals are willing and able to realise plans of action and bring about change.

Hence, 'information', 'motivation' and 'realization' seemed to serve as potential mediating pathways by which M\&M drives learning and improvement (table 2). These pathways could also affect each other as, for example, information can motivate by increasing sense of urgency, which may ultimately enhance realisation efforts.

\section{DISCUSSION}

This qualitative study identified 57 different barriers and facilitators to successful M\&M practice perceived by healthcare professionals, together covering 17 themes. Many factors concerned organisational aspects, but others related to the individual or team level, such as personal motivation or group dynamics. All factors affected whether participants are (1) motivated to 
participate and take action, (2) well-informed to identify targets and plans for improvement, and (3) willing and able to realise plans, representing the mediating pathways to M\&M-based learning and improvement.

An important strength of this study lies in the qualitative approach, yielding nuanced insights that quantitative assessments cannot reveal. To illustrate, qualitative analyses revealed the complexity of various factors, such as hierarchy or team spirit, which appeared to have both positive and negative effects at the same time. Moreover, data saturation was achieved and many factors and pathways described in the study appeared to closely relate to more general frameworks and theories of learning and change. An important limitation is the single-centre design of this study. The findings may particularly be representative of teaching hospitals as interviewees worked at an academic hospital and their prior M\&M experience was mostly at other teaching hospitals. However, qualitative research does not pursue generalisability, but rather aims to explore and develop a deeper understanding of a phenomenon of interest. As interviewees worked in surgery, these findings may not be fully representative of all medical specialties that practise M\&M. Additional qualitative research is required to reveal whether the same facilitators and barriers apply to other specialties. This is likely the case, as the generic mechanisms by which clinicians learn and improve through these conferences will be more similar. Research on M\&M in other settings, such as paediatrics and psychiatry, highlight similar success factors, including resources (ie, time and staff), ${ }^{33} 34$ leadership buy-in and presence, ${ }^{3435}$ input from all staff levels, ${ }^{33-36}$ and loop closure. ${ }^{33} 35$ Furthermore, in a previous study, we found that departments with great variation in M\&M practice shared the same expectations and challenges for M\&M. ${ }^{29}$ Moreover, the study findings appeared to fit well within the more general frameworks for learning and improvement in healthcare (online supplementary appendix 3).

Table 3 Recommendations for successful M\&M practice based on identified facilitators and barriers, and mediating pathways for M\&M-based learning and improvement*

\section{Recommendation}

\section{Urgency}

Select topics relevant to the audience and demonstrate a sense of urgency.

\section{Information}

Maximise informativeness and attractiveness of presentations.

\section{Planning Be explicit in terms of action items and follow-up.}

4. Motivation

Motivate participants through interactivity and feedback.

\section{Anticipation}

Consider feasibility of actions, and anticipate and counter problems.

\section{Input}

Draw on collective expertise of participants.

\section{Receptivity \\ Cultivate an open mindset, receptive to all input and opportunities.}

\section{Setting}

Consider M\&M meetings in specialist settings.

\section{Resources \\ Dedicate time and staff to M\&M practice and ensuing plans for improvement.}

\section{Data}

Dedicate time and staff to M\&M practice and ensuing actions for improvement.

\section{Further details (related themes in table 1)}

Ensure topics are applicable to one's own practice, clinically significant and accompanied by a sense of urgency, for example, by supporting presentations with (local) data on incidences and harm $(1,4,13)$.

Use well-prepared presenters, engagement of those involved in cases, and fixed presentation formats including case details, literature, local/ benchmark data, as well as system-level and soft/human factors $(2,3,6)$.

Determine who will do what, when and how, with a plan for follow-up and re-evaluation $(5,10,13)$.

Ensure that participants are motivated, for example, by using moderators to promote interactivity and 'close the loop' on prior actions through evaluation and feedback (6, 10-14).

\section{Anticipate and plan how to counter problems with realisation}

and sustaining of actions, for example, due to complexity, lack of empowerment or engagement of all staff involved, or staff turnover $(4,7,10)$.

Ensure presence and input from all involved in care processes, for example, by actively inviting comments from experts, juniors or other disciplines (7, 9-11).

Emphasise that input of all involved in care is essential and valued as such, and underline the need to be sensitive to 'weak signals' that may signal opportunities for improvement $(7,9-13)$.

In meetings on the subspecialty or multidisciplinary level ('integrated care'), participants may be more informed and in control as topics are more closely related to their daily practice $(8,9,13,15)$.

Consider blocking time for attendance but also preparation and realisation of actions, and consider use of a dedicated committee or staff to implement plans that ensue from $\operatorname{M\& M}(6,10,15)$.

Ensure that data collection and monitoring systems are accessible to allow assessment of local performance, benchmarking against others and reevaluation of prior plans for improvement $(14,17)$.

${ }^{*}$ There is no hierarchical order in this list. Numbers, how recommendations relate to earlier published frameworks for improvement in healthcare and to mediating pathways, are depicted in online supplementary appendix 3.

$\mathrm{M} \& \mathrm{M}$, morbidity and mortality conference. 


\section{Comparison with existing literature}

While M\&M practice has often been subject of study, this is, to our knowledge, the first qualitative study of M\&M success factors. The present study adds novel insights into the roles of various individual-level and social-level factors, perceived as barriers, facilitators or both simultaneously (table 1) - an example being 'team spirit', which was perceived as a potential facilitator as well as barrier to openly voicing one's opinions or concerns at M\&M. Thus far, individual-level or team-level factors have received scant attention in the M\&M literature, with the exception of the importance of 'a blame-free culture'. 25122037 This study confirms the importance of a safe environment, but also provides leads about what the desired culture or 'mindset' for M\&M encompasses. It seems that M\&M should elicit input from all participants, ${ }^{10} 1516$ and truly value such input from all corners. In other words, attention needs to be given to both the sender and receiver end to harness a truly open mindset at the conference. The value of input from other disciplines was appreciated by interviewed professionals, but multidisciplinarity was also perceived as a potential threat to the open environment that is so important for M\&M. This finding adds nuance to previous studies advocating for multidisciplinary M\&M, expecting only positive effects. ${ }^{10}{ }^{37-39}$

This study revealed three mediating pathways by which M\&M may successfully drive learning and improvement, which were related to information, motivation and realisation (table 2). While the role of motivation has received little attention in prior M\&M research, more general publications about organisational learning or improvement have stressed the important role of individual and team factors, such as motivation. ${ }^{21-24}$ After all, leadership can create strategies and improvement plans, but this will be insufficient without commitment and support of front-line staff-'culture eats strategy for breakfast'. ${ }^{24} 4041$ Pathways to M\&M success described in this study appeared to closely relate to more general frameworks for improvement and implementation in healthcare (online supplementary appendix 3). We attempted to translate the findings of this qualitative study to actionable recommendations, enlisted in table 3, targeting one or more of the described pathways to M\&M success. Some of these recommendations have been reported in prior M\&M studies, such as using local data ${ }^{42}{ }^{43}$ and extensive planning, ${ }^{10}$ but others more closely relate to learning behaviour literature, such as sense of urgency, motivation and being receptive to new ideas. ${ }^{21232441}$

\section{Implications for M\&M practice}

The recommendations formulated based on the study findings address some aspects of M\&M organisation, but also aim to target challenges at the level of the (individual) professionals (table 3). Various complexities embedded in healthcare culture may complicate M\&M practice, one of which is working with colleagues with different hierarchical or expertise levels. These professional boundaries might be overcome by promoting the desired mindset for M\&M. As with the "culture of shame and blame', which used to be infamous for its presence at M\&M, these issues could be targeted with, for example, moderators and local leadership, guided by principles of Just Culture. ${ }^{44}$ As mentioned in the interviews, seniors or leaders can model desired behaviour and attitudes at M\&M, by openly discussing personal errors and addressing the emotional impact. This is confirmed by, to our knowledge, the only other qualitative study of M\&M, conducted in internal medicine, which described this type of role-modelling at the conference. ${ }^{46}$ For example, the conference could start with framing the purpose as collegial and non-blaming, as used in recently developed novel formats for M\&M. ${ }^{33-35}$

An important question for future research appears to be how to motivate and engage all participants to receive the necessary input and support to actually improve clinical practice. Interviews reflected the paradoxical nature of hierarchy in this respect, as this can both help and hurt staff's motivation and support. Another solution may be to organise M\&M in smaller, focused settings, such as subspecialties ${ }^{15}$ or integrated care. Interviewees also perceived motivational effects of reviewing local or benchmark data and follow-up of actions from prior conferences, which could be incorporated into M\&M practices to motivate participants and demonstrate the value of M\&M. ${ }^{50}$ More time for feedback and assessment of prior initiatives would mean that fewer topics can be discussed at M\&M or that extra time needs to be made available, but this would both be worthwhile considering the expected positive effects on achieving sustainable improvements.

\section{CONCLUSION}

This study enhanced understanding of the factors influencing M\&M-based learning and improvement, and the pathways by which this occurs. Many factors were related to the individual or team rather than how M\&M is organised. These insights may be used to improve M\&M practices and provide a framework for further study on determinants of M\&M success. Future research is warranted to investigate success factors for M\&M, and specifically the extent to which these are transferable to other settings, in order to design a universally applicable best practice for M\&M.

Acknowledgements We like to show our gratitude to all faculty and residents willing to reflect and express their opinions so openly during the interviews. We thank Judith van Grafhorst for her valuable assistance with the interview transcriptions and analyses.

Contributors All authors (MSdV, JFH, PJM) contributed to conception and design of this study. MSdV generated, analysed and interpreted the data, wrote the first draft and revised drafts. JFH revised the draft papers. PJM supervised data analysis and 
interpretation, and revised the draft paper. All authors have seen and approved the final draft. All authors received access to all the anonymised data in the study and take responsibility for the integrity and the accuracy of the data analysis. PJM is the guarantor

Funding This work was supported by a combined unrestricted grant from the Board of Directors of the Leiden University Medical Centre, Vogelgezang Foundation (no. 1519-01), Leiden University Fund (no. 5265/12-11-15) and the Michäel-Van Vloten Surgery Fund. Funders had no role in the study design, data analysis and interpretation, writing of the report, or in the decision to submit the article for publication.

\section{Competing interests None declared.}

Ethics approval Not required under Dutch National Law. All interviewed professionals verbally consented to participate in this study.

Provenance and peer review Not commissioned; externally peer reviewed.

Data sharing statement Qualitative data generated and analysed in the current study are verbatim transcripts (in Dutch) and are not publicly available to protect the privacy of interviewees.

Open Access This is an Open Access article distributed in accordance with the Creative Commons Attribution Non Commercial (CC BY-NC 4.0) license, which permits others to distribute, remix, adapt, build upon this work non-commercially, and license their derivative works on different terms, provided the original work is properly cited and the use is non-commercial. See: http://creativecommons.org/ licenses/by-nc/4.0/

(C) Article author(s) (or their employer(s) unless otherwise stated in the text of the article) 2017. All rights reserved. No commercial use is permitted unless otherwise expressly granted.

\section{REFERENCES}

1. Xiong $X$, Johnson $T$, Jayaraman $D$, et al. At the crossroad with morbidity and mortality conferences: lessons learned through a narrative systematic review. Can J Gastroenterol Hepatol 2016;2016:1-11.

2. Orlander JD, Barber TW, Fincke BG. The morbidity and mortality conference: the delicate nature of learning from error. Acad Med 2002;77:1001-6.

3. Aboumatar HJ, Blackledge CG, Dickson C, et al. A descriptive study of morbidity and mortality conferences and their conformity to medical incident analysis models: results of the morbidity and mortality conference improvement study, phase 1. Am J Med Qual 2007;22:232-8

4. Nasca TJ, Philibert I, Brigham T, et al. The next GME accreditation system--rationale and benefits. N Engl J Med 2012;366:1051-6.

5. Sacks GD, Lawson EH, Tillou A, et al. Morbidity and mortality conference 2.0. Ann Surg 2015;262:228-9.

6. Macrae C. The problem with incident reporting. BMJ Qual Saf 2016;25:71-5.

7. Cooke DL, Dunscombe PB, Lee RC. Using a survey of incident reporting and learning practices to improve organisational learning at a cancer care centre. Qual Saf Heal Care 2007;16:342-8.

8. Ginsburg LR, Chuang YT, Norton PG, et al. Development of a measure of patient safety event learning responses: Patient safety and public health. Health Serv Res 2009;44:2123-47.

9. Vincent $C$. Understanding and responding to adverse events. N Engl $J$ Med 2003;348:1051-6.

10. Bal G, Sellier E, Tchouda SD, et al. Improving quality of care and patient safety through morbidity and mortality conferences. J Healthc Qual 2014;36:29-36.

11. François P, Prate F, Vidal-Trecan G, et al. Characteristics of morbidity and mortality conferences associated with the implementation of patient safety improvement initiatives, an observational study. BMC Health Serv Res 2016;16:35.

12. Bechtold ML, Scott S, Dellsperger KC, et al. Educational quality improvement report: outcomes from a revised morbidity and mortality format that emphasised patient safety. Postgrad Med $\mathrm{J}$ 2008;84:211-6.

13. Gordon L. Gordon's guide to the surgical morbidity and mortality conference. Philadelphia, PA, USA: Hanley and Belfus, 1994.

14. Murayama KM, Derossis AM, DaRosa DA, et al. A critical evaluation of the morbidity and mortality conference. Am J Surg 2002;183:246-50.

15. Gore DC. National survey of surgical morbidity and mortality conferences. Am J Surg 2006;191:708-14.
16. Risucci DA, Sullivan T, DiRusso S, et al. Assessing educational validity of the morbidity and mortality conference: a pilot study. Curr Surg 2003;60:204-9.

17. Lecoanet A, Vidal-Trecan G, Prate F, et al. Assessment of the contribution of morbidity and mortality conferences to quality and safety improvement: a survey of participants' perceptions. BMC Health Serv Res 2016;16:176.

18. Harbison SP, Regehr G. Faculty and resident opinions regarding the role of morbidity and mortality conference. Am J Surg 1999;177:136-9.

19. Kim MJ, Fleming FJ, Peters JH, et al. Improvement in educational effectiveness of morbidity and mortality conferences with structured presentation and analysis of complications. J Surg Educ 2010;67:400-5.

20. Flynn-O'Brien KT, Mandell SP, Eaton EV, et al. Surgery and medicine residents' perspectives of morbidity and mortality conference: an interdisciplinary approach to improve ACGME core competency compliance. J Surg Educ 2015;72:e258-66.

21. Chuang YT, Ginsburg L, Berta WB. Learning from preventable adverse events in health care organizations: development of a multilevel model of learning and propositions. Health Care Manage Rev 2007;32:330-40.

22. Grol R, Wensing M. What drives change? Barriers to and incentives for achieving evidence-based practice. Med J Aust 2004;180:S57-60.

23. Gibson C, Vermeulen F. A healthy divide: subgroups as a stimulus for team learning behavior. Adm Sci Q 2003;48:202-39.

24. Carroll JS, Edmondson AC. Leading organisational learning in health care. Qual Saf Health Care 2002;11:51-6.

25. Dicicco-Bloom B, Crabtree BF. The qualitative research interview. Med Educ 2006;40:314-21.

26. Francis $\mathrm{JJ}$, Johnston $\mathrm{M}$, Robertson $\mathrm{C}$, et al. What is an adequate sample size? Operationalising data saturation for theory-based interview studies. Psychol Health 2010;25:1229-45.

27. O'Brien BC, Harris IB, Beckman TJ, et al. Standards for reporting qualitative research. Acad Med 2014;89:1245-51.

28. Kievit J, Krukerink M, Marang-van de Mheen PJ. Surgical adverse outcome reporting as part of routine clinical care. Qual Saf Health Care 2010;19:e20.

29. de Vos MS, Marang-van de Mheen PJ, Smith AD, et al. Toward best practices for surgical morbidity and mortality conferences: a mixed methods study. J Surg Educ 2017;15.

30. Braun V, Clarke V. Using thematic analysis in psychology. Qual Res Psychol 2006;3:77-101.

31. Saldaña J. The coding manual for qualitative researchers. London, UK: SAGE Publications Ltd, 2009.

32. de Vos MS, Smith AD, Shimizu N, et al. Surgical quality improvement book (chapter 5): morbidity and mortality conference: a weekly conference Ddesigned to improve surgical quality. http://www. brighamandwomens.org/Departments_and_Services/surgery/ qiChapter5.aspx?sub=0 (accessed 24 Jul 2017).

33. Cromeens B, Brilli R, Kurtovic K, et al. Implementation of a pediatric surgical quality improvement (QI)-driven M\&M conference. J Pediatr Surg 2016;51:137-42.

34. Wasser T, Grunschel BD, Stevens A, et al. Transforming systems of care through a novel resident-led approach to morbidity and mortality conferences. Acad Psychiatry 2016;40:893-7.

35. Gerstein WH, Ledford J, Cooper J, et al. Interdisciplinary quality improvement conference: using a revised morbidity and mortality format to focus on systems-based patient safety issues in a VA hospital: design and outcomes. Am J Med Qual 2016;31:162-8.

36. Frey B, Doell C, Klauwer D, et al. The morbidity and mortality conference in pediatric intensive care as a means for improving patient safety. Pediatr Crit Care Med 2016;17:67-72.

37. Kwok ESH, Calder LA, Barlow-Krelina E, et al. Implementation of a structured hospital-wide morbidity and mortality rounds model. BMJ Qual Saf 2017;26:439-48.

38. Kauffmann RM, Landman MP, Shelton J, et al. The use of a multidisciplinary morbidity and mortality conference to incorporate ACGME general competencies. J Surg Educ 2011;68:303-8.

39. Deis JN, Smith KM, Warren MD, et al. Advances in patient safety: new directions and alternative approaches (Vol. 2: culture and redesign). http://www.ncbi.nlm.nih.gov/pubmed/21249895 (accessed 23 Mar 2017)

40. Dixon-Woods M, Leslie M, Tarrant C, et al. Explaining matching Michigan: an ethnographic study of a patient safety program. Implement Sci 2013;8:70.

41. Dixon-Woods M, McNicol S, Martin G. Ten challenges in improving quality in healthcare: lessons from the Health Foundation's programme evaluations and relevant literature. BMJ Qual Saf 2012;21:876-84. 
42. Hamby LS, Birkmeyer JD, Birkmeyer C, et al. Using prospective outcomes data to improve morbidity and mortality conferences. Curr Surg 2000;57:384-8.

43. Hutter MM, Rowell KS, Devaney LA, et al. Identification of surgical complications and deaths: an assessment of the traditional surgical morbidity and mortality conference compared with the American College of Surgeons-National Surgical Quality Improvement Program. J Am Coll Surg 2006;203:618-24.
44. Martinez W, Lehmann LS, Hu YY, et al. Processes for identifying and reviewing adverse events and near misses at an academic medical center. Jt Comm J Qual Patient Saf 2017;43:5-15.

45. Dekker S. Just culture: restoring trust and accountability in Your organization. Taylor \& Francis Ltd, 2016.

46. Kuper A, Nedden NZ, Etchells E, et al. Teaching and learning in morbidity and mortality rounds: an ethnographic study. Med Educ 2010;44:559-69. 